\title{
The status of the CMS pixel upgrade detector
}

\author{
Ferdinando Giordano* \\ University of California Riverside \\ E-mail: Ferdinando.Giordano@cern.ch
}

The present CMS pixel detector was designed for a maximum luminosity of $1 \times 10^{34} \mathrm{~cm}^{-2} \mathrm{~s}^{-1}$. Following the Phase 1 upgrade of the LHC, the peak luminosity is foreseen to reach $2 \times 10^{34} \mathrm{~cm}^{-2} \mathrm{~s}^{-1}$. Due to the radiation damage and large data losses in the readout chip the present pixel system must be replaced by a new one in the extended year-end technical stop 2016/2017. The current status of the CMS pixel detector upgrade project will be presented. The new features of the proposed detector that will be discussed are the ultra-light mechanical design with four barrel layers and three end-cap disks, the digital readout chip with higher rate capability, and the new cooling system.

36th International Conference on High Energy Physics

4-11 July 2012

Melbourne, Australia

* Speaker.

${ }^{\dagger}$ on behalf of the CMS Collaboration. 


\section{Introduction}

The pixel detector is a key element of CMS and maintaining its performance is of fundamental importance for the whole CMS experiment. Since its design, an installation scheme that allows replacement even in short yearly technical shutdowns was considered [1]. A replacement was already foreseen in case of performance degradation because the pixel detector is subject to intense radiation.

Given the current LHC schedule to further increase its instantaneous luminosity to $2 \times 10^{34} \mathrm{~cm}^{-2} \mathrm{~s}^{-1}$ and the predicted radiation damage, a replacement of the current detector with an improved version with a new 4-layers / 3-disks system is planned for 2016/2017.

The basic concept of the pixel detector upgrade [2] foresees improvements in tracking quality, impact parameter resolution and rate handling capability. However, building the new detector is not only constrained by technical feasibility but also by the present detector and the CMS experiment facility. Moreover potential schedule conflicts with LHC and CMS must be considered. The current plan is to change the pixel detector during a technical stop in 2016/2017, using many of the components already in place for the current detector. This includes power lines, cooling pipes, and read-out fibers which pass through the whole body of CMS.

\section{Improvement of tracking}

The goal of the upgrade detector is to improve, or at least maintain, the performance of the current detector in the high luminosity environment. The pixel detector provides essential information for track reconstruction. Enhanced performance of the pixel tracks will improve tracking efficiency, fake track rejection, vertex finding, $b$-tagging, and high-level trigger capabilities as well.

In order to achieve better performance the new pixel detector will feature an extra barrel layer and end-cap disk. In the new design each track will normally have four hits up to $|\eta|=2.5$ versus the present three hits per track (see figure 1). The new design has a smaller inner radius for the innermost layer to improve vertex finding and reconstruction, thus enhancing $b$-tagging performance.
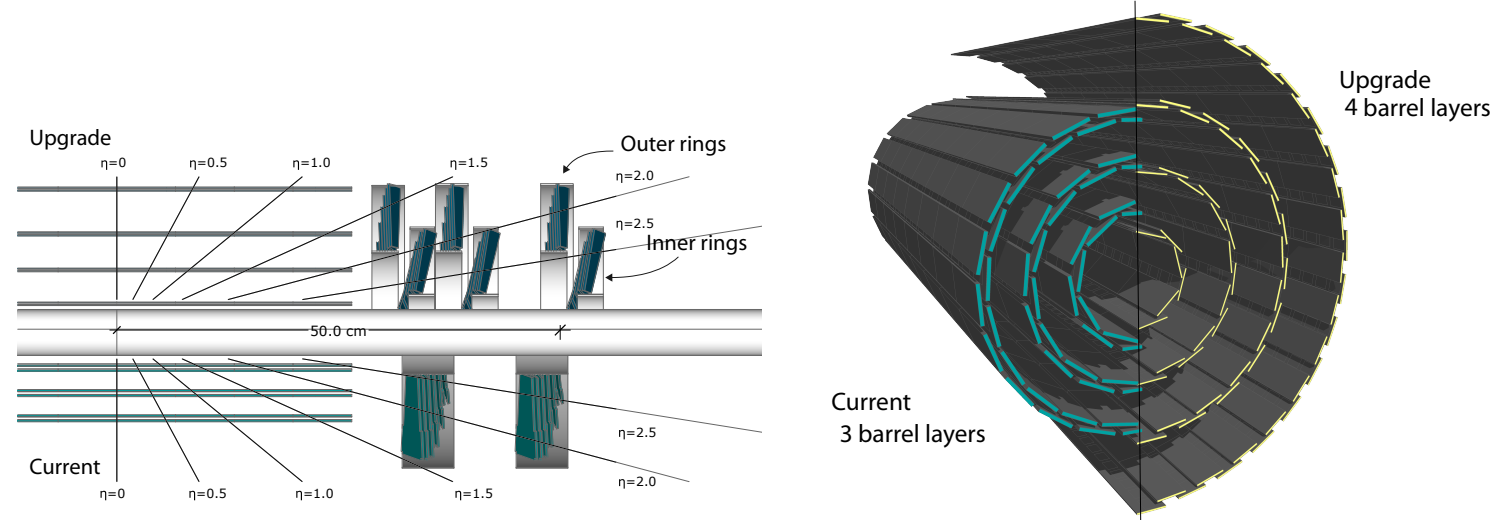

Figure 1: Left: Conceptual layout comparing the different layers and disks in the current and upgrade pixel detectors. Right: Transverse-oblique view comparing the pixel barrel layers in the two detectors.

To avoid increasing the total mass of the detector in proportion to the number of layers in the new design, all components were examined with the goal of lowering their mass. The mass of the 
new concept, even with the extra layer, is significantly lower than the current detector: the barrel (defined as $|\eta|<2.16$ ) goes from $16801 \mathrm{~g}$ to $6686 \mathrm{~g}$, the forward pixel $(|\eta|<2.50)$ from $8582 \mathrm{~g}$ to $7040 \mathrm{~g}$. As we can see in figure 2, the lighter design (black dots) has a significant reduction in radiation length and nuclear interaction length compared to the current one (green histogram). This reduction is achieved thanks to lighter materials in the mechanical design, a new cooling system and modified placement of many of the service components now located outside the tracking region at $|\eta|>2.50$.
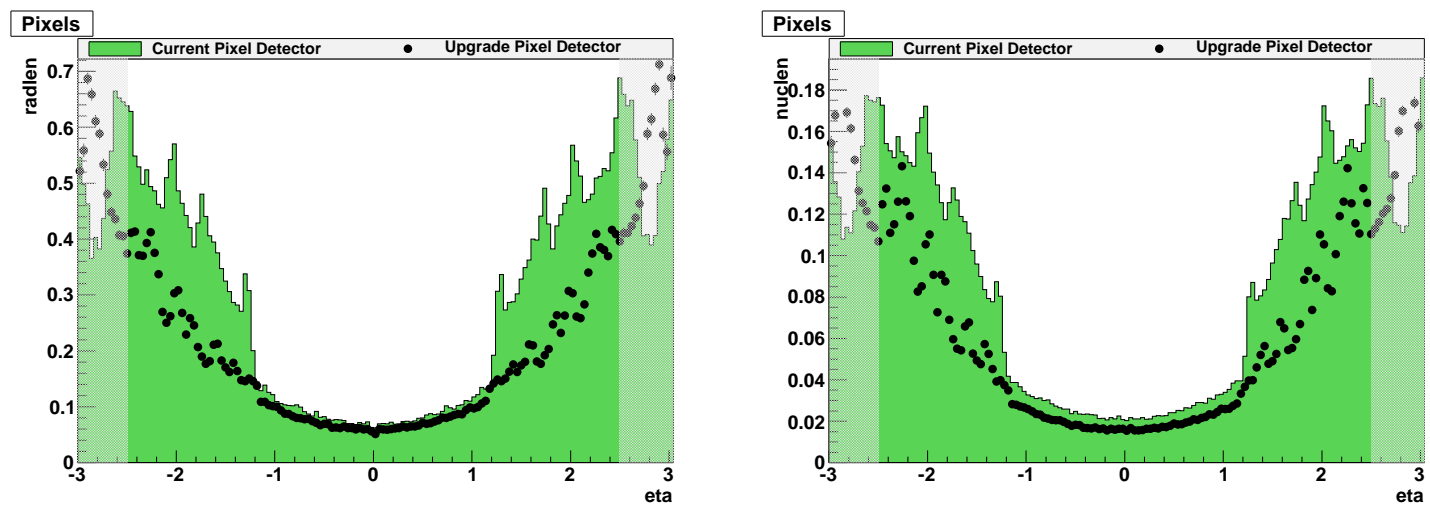

Figure 2: The amount of material in the pixel detector shown in units of radiation length (left), and in units of nuclear interaction length (right) as a function of $\eta$; this is given for the current pixel detector (green histogram), and the Phase 1 upgrade detector (black points). The shaded region at high $|\eta|$ is outside the region for track reconstruction.

As mentioned above, a major reduction in mass is achieved going from the liquid $\mathrm{C}_{6} \mathrm{~F}_{14}$ cooling used in the present detector to a two-phase $\mathrm{CO}_{2}$ system. Evaporative cooling is an appealing technology, in particular for tracking detectors with high power density, as it provides high cooling efficiency with minimal amount of material. The choice of $\mathrm{CO}_{2}$ as refrigerant is particularly advantageous, because of its excellent thermodynamical properties: such as small viscosity, high heat transfer, high latent heat and low liquid/vapour density ratio that allow the use of very small pipes (typically $16 / 18 \mathrm{~mm}$ inner diameter) which can stand the high operational pressure (about $20 \mathrm{bar}$ ), and because of its low density and radiation hardness. In addition $\mathrm{CO}_{2}$ is substantially cheaper than fluorocarbon refrigerants and has much lower impact on the environment.

The new cooling system consists of two separate systems, one for the barrel and one for the disks, that can be operated independently at different temperatures. Each of them is also capable of cooling down the whole detector to ensure both operational freedom and redundancy in case of failures.

Figure 3 shows the tracking performance improvement of the upgrade detector over the current detector. Different scenarios have been simulated starting from no pileup (blue squares), LHC design luminosity (red dots), and high luminosity scenario with $25 \mathrm{~ns}$ (black diamonds) and $50 \mathrm{~ns}$ bunch spacing (brown triangles). The number of underlying events increases from 0 to 100 and the upgrade detector is able to maintain its performance in terms of efficiency and fake track suppression for almost the whole considered range, while the current detector quickly loses performance at high pileup. 

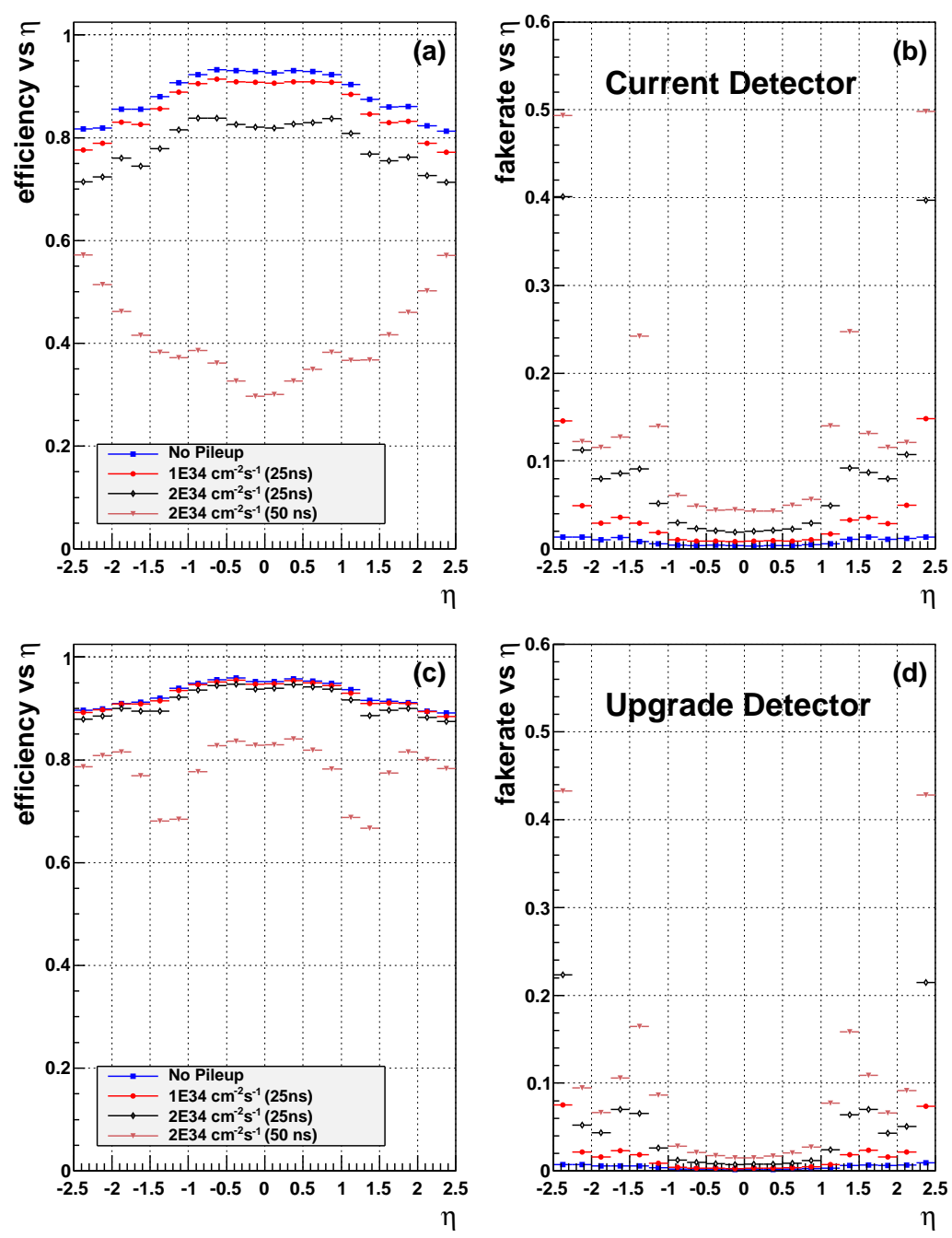

Figure 3: Tracking efficiency $(\mathrm{a}, \mathrm{c})$ and fake rate $(\mathrm{b}, \mathrm{d})$ for a $t \bar{t}$ sample as a function of track $\eta$, for the current detector $(\mathrm{a}, \mathrm{b})$ and the upgrade pixel detector $(\mathrm{c}, \mathrm{d})$. Results are shown for zero pileup (blue squares), an average pileup of 25 (red dots), an average pileup of 50 (black diamonds), and an average pileup of 100 (brown triangles) with ROC data loss simulation expected at the given luminosities.

\section{Vertex resolution and $b$-tagging}

The new CMS beam-pipe, with a smaller radius and the central part made of pure beryllium, allows reduction of the radius of the first layer of the pixel barrel from $44 \mathrm{~mm}$ to $30 \mathrm{~mm}$. Together with improved tracking quality as described above this brings significant improvements in the vertex resolutions (see fig. 4).

In figure 5 we show a direct comparison of $b$-tagging performance between current and future geometries without pileup and in presence of 50 pileup events. The new geometry has better performance in terms of $b$-tag efficiency for a given purity selection or a better $c /$ light-jets rejection for the same $b$-tag efficiency. Moreover, the new geometry will assure the same performance as the present geometry even at much higher pileup. 

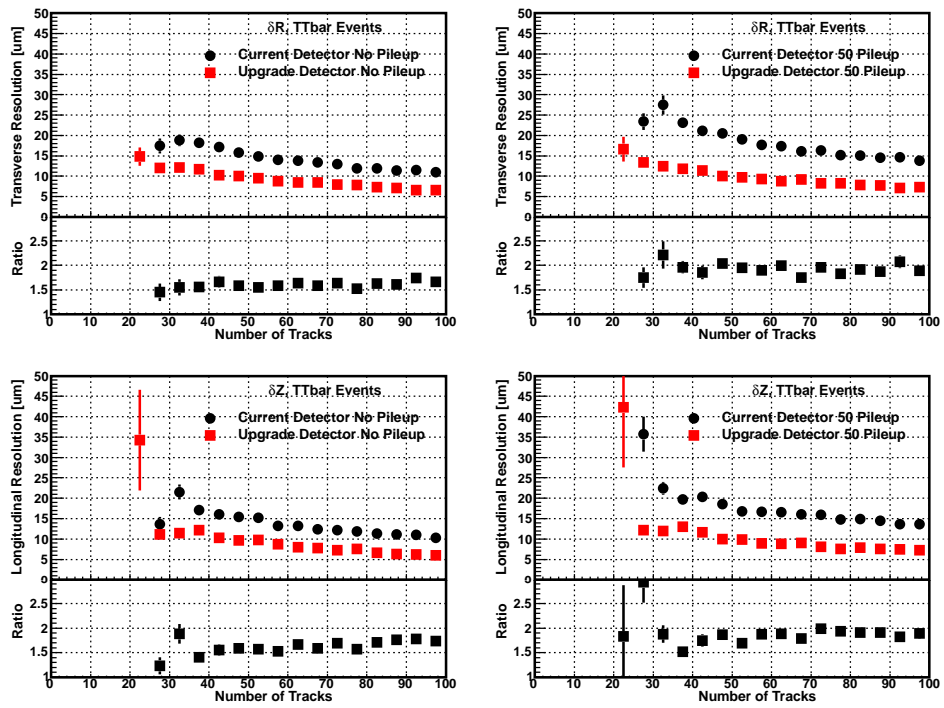

Figure 4: Transverse (top) and longitudinal (bottom) primary vertex resolutions as a function of the number of tracks in the vertex for a $t \bar{t}$ sample with (left) zero pileup, and (right) with an average pileup of 50. The resolutions are shown for the current pixel detector (black circles) and the Phase 1 upgrade detector (red squares). The lower part of each plot shows the ratio of the current detector resolution to the upgrade resolution.
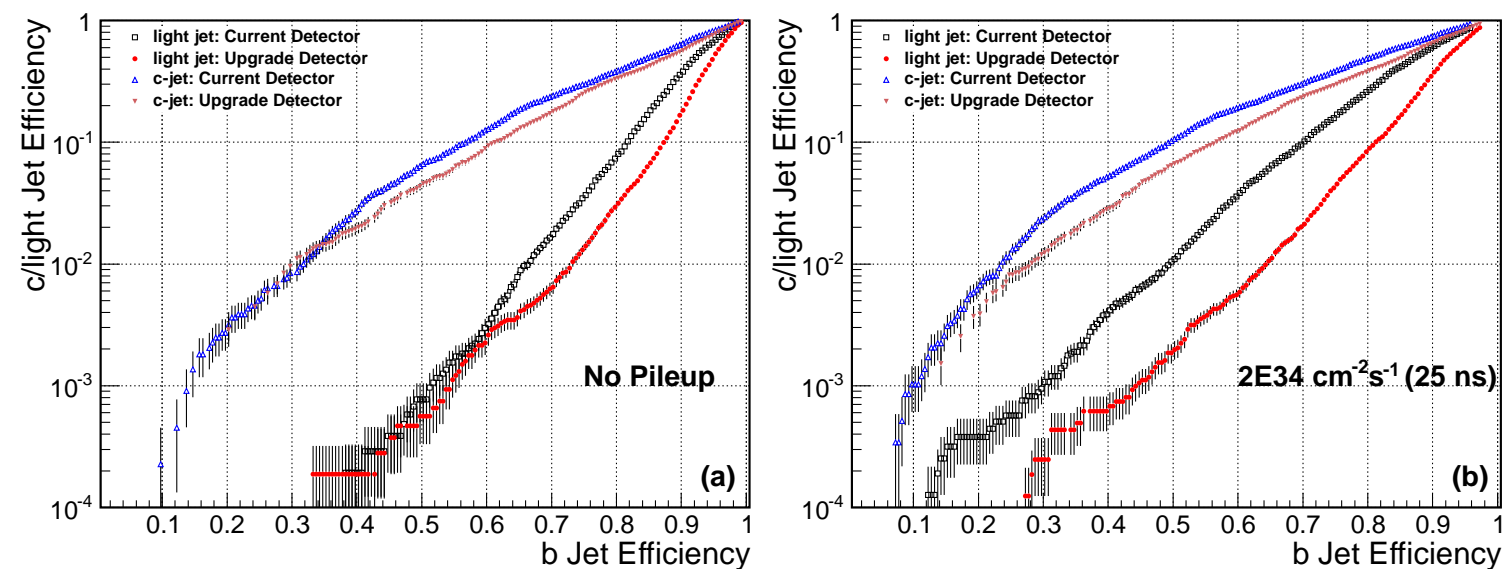

Figure 5: Performance of the Combined Secondary Vertex $b$-tagging algorithm for jets with $\mathrm{p}_{\mathrm{T}}>30 \mathrm{GeV}$ in a $t \bar{t}$ sample with (a) zero pileup, and (b) an average pileup of 50. The performance for the current detector is shown by the open points while the solid points are for the upgrade detector. The triangular points are for $c$-jets while the circle and square points are for light quark jets.

\section{Improved readout electronics}

The current readout chain has been designed for operation at $25 \mathrm{~ns}$ bunch spacing and instantaneous luminosity up to $10^{34} \mathrm{~cm}^{-2} \mathrm{~s}^{-1}$. At design luminosity the data-loss will be only a few percent. However, the current LHC schedule to increase the luminosity up to $2 \times 10^{34} \mathrm{~cm}^{-2} \mathrm{~s}^{-1}$, and potentially use a $50 \mathrm{~ns}$ bunch space will result in a significant enhancement of data rates. Consequently, due to limitations of the readout chip (ROC) architecture and output data bandwidth in the current 
detector, this would result in a dramatic drop in efficiency to $50 \%$ or less for the innermost layer.

In order to provide a reliable readout chain, with high pileup, a new ROC was designed. Major improvements include the replacement of the analog data transfer by a digital readout and the increase of the length of local data buffers. Digitalization is performed by an on-chip 8 bit ADC at a speed of $80 \mathrm{MHz}$. The data are sent to the Token Bit Manager (TBM) at $160 \mathrm{MHz}$, then the frequency is doubled once more and via optical fibers the signal is sent to the Frond End Driver (FED) located in the service cavern. Thanks to the new system the data throughput per fiber will be doubled, reaching a good safety margin for the outer layers (2-4), while for the innermost the number of fibers per module will increase from two to four, resulting in an overall factor of 4 over the present layer 1 throughput to ensure the same safety margin.

\section{Consequences for chip powering}

The power system of the new detector will also have to be modified, but due to access limitations and space constraints as well as in order to contain cost and time both the existing cables and power supplies will be reused. The Phase-1 detector with the extra layers needs more power to be operated and the current power supplies and cables cannot cope with the larger current that would be consumed by the new detector because of large heat losses.

This problem can be solved using DC-DC converters close to the detector which would provide the low voltage needed, reducing cable losses. This set-up has the advantage that only minor modifications are needed to the current CAEN power supplies and all the current cabling can be reused. The needed DC-DC converters have already been tested and been proven to give the required performance to operate the Pixel detector; in particular good efficiency, shielding and radiation hardness.

\section{Conclusion}

The replacement of the current Pixel detector will give many benefits to the whole CMS detector. The major enhancement comes from the extra layer and the capability to handle the higher occupancy in the detector with high pileup, thus keeping high efficiency and good fake track rejection. At the same time a significant reduction in the material budget of the Phase- 1 detector is achieved trough improved mechanical design and by moving to the $\mathrm{CO}_{2}$ cooling system.

The readout electronics will be replaced to ensure low dead time even in the highly demanding scenarios of $2 \times 10^{34} \mathrm{~cm}^{-2} \mathrm{~s}^{-1}$ luminosity with bunch crossing times longer than $25 \mathrm{~ns}$.

The installation of the new system is planned to start in 2013 during the long shutdown with the $\mathrm{CO}_{2}$ cooling system and the new beam-pipe, while the new detector can be installed in an extended technical stop around 2016/2017.

\section{References}

[1] The CMS Collaboration, The Tracker System Project, Technical Design Report, CERN/LHCC 98-6, Geneva 1998.

[2] The CMS Collaboration, CMS Technical Design Report for the Pixel Detector Upgrade, CERN-LHCC-2012-016, Geneva 2012. 